\title{
Comparison of Total Phenolic Contents and Antioxidant Activities of Centella asiatica Extracts Obtained by Three Extraction Techniques
}

\author{
W.N.H.W. Zainal ${ }^{* 1}$, F.R. Musahib ${ }^{1}$, and N.S. Zulkeflee ${ }^{1}$ \\ ${ }^{1}$ Faculty of Chemical and Process Engineering Technology, Universiti Malaysia Pahang, 26300 Kuantan, \\ Pahang, Malaysia
}

Email: wannurulhuda@ump.edu.my*

\begin{abstract}
Through different extraction techniques, the potential of Centella asiatica as a natural source of antioxidant was investigated. The $C$. asiatica aqueous extracts were obtained via infusion, decoction and ultrasound-assisted extraction techniques. The effects of different extraction techniques were studied on the extraction yield, total phenolic contents and antioxidant activity. The total phenolic contents of the extracts and antioxidant activity were examined using the Folin-Ciocalteu's reagent and 2,2-diphenyl-1-picrylhydrazyl (DPPH) free radical scavenging activity assay, respectively. Results indicated that the UAE exhibited the highest extraction yield, highest total phenolic contents, as well as highest antioxidant activity. The yield of the extracts increased in the order of infusion < decoction < UAE which were $18.2 \%, 23.6 \%$ and $25.4 \%$, respectively. All extraction techniques had a significant effect $(p<0.05)$ on the total phenolic contents and antioxidant activity of $C$. asiatica extracts. The total phenolic contents ranged from $3.42 \pm 0.030$ to $8.32 \pm 0.105 \mathrm{mg} \mathrm{GAE} / \mathrm{g}$ dry extract while the antioxidant activity was in the range of 75 to $86 \%$. This study confirms that $C$. asiatica has the potential to be a good resource for the future development of natural antioxidant. In addition, extraction via UAE can be an ideal technique to obtain phytochemical-rich extracts from medicinal plants.
\end{abstract}

Keywords - C. asiatica, total phenolic contents, extraction, antioxidant activity, ultrasound-assisted extraction

\section{INTRODUCTION}

The use of synthetic antioxidants compounds has been restricted by legislation due to doubts about their toxic and carcinogenic effects. Synthetic antioxidants like butylated hydroxytoluene (BHT) and butylated hydroxyanisole (BHA) are reported to have toxic effects on the lungs and induce the formation of tumours [1]. Thus, increasing attention has been directed toward natural and safer antioxidants that could be isolated from natural sources and efficiently clear free radicals. Furthermore, the isolation of bioactive compounds from natural sources requires less volume of solvents and produces fewer toxic by-products. Plants have been reported as a potential source of natural antioxidant compounds. Phenolic and flavonoids are commonly known as the most common phytochemical molecules with antioxidant properties present in plants [2,3]. A large number of plants like fruits and vegetables have been explored for their antioxidant potential such as bhut jolokia, peach, apples, cranberry, baby Chinese cabbage, broccoli, celery, chives, eggplant, mustard, onion, red pepper, spinach, and tomato.

Typically, the biologically active compounds in plants are present in low concentration. Prior to determining the bioactive compounds, extraction of compounds from plant materials is an important step. Extraction can be defined as a separation process where the distribution of analyte between two immiscible phases is based on the appropriate distribution coefficient [4]. Traditional extraction process using organic solvents often suffers from low extraction yields, long extraction times and has residual toxic organic solvents in final products. The residual of organic solvents in the extract can cause serious health problems if the extracts are taken into the human body [5]. Hence, it is more practical and safer 
for the consumer if the plant material is extracted using water [6]. After extraction solvent has been selected, the most appropriate extraction method should be carefully selected considering its practicality, efficacy and time consumption that is appropriate to conduct the extraction of medicinal plants. There are various extraction techniques have been used, namely conventional techniques such as infusion, decoction and Soxhlet extraction, and non-conventional techniques such as ultrasound-assisted and microwave-assisted [7-10]. Some studies have reported variations in the number of biologically active compounds and their biological activity obtained by different extraction techniques [3,4,7,8]. Moreover, process variables also should be optimized for the most effective isolation of target constituents as they also influenced the number of biological compounds [10].

C. asiatica or pegaga is one of the medicinal herbs that have been used for thousands of years all over the world. It is easily grown and found in most tropical and subtropical countries. This medicinal plant has been used since ancient times for various medicinal and cosmetic purposes. Based on Ayurveda, $C$. asiatica is one of the main herbs used for revitalizing nerves and brain cells [11]. It has also been used to promote longevity, lower high blood pressure and improve memory. In addition to its use in the medicinal plant, it is also eaten raw as a salad, cooked as a dish, and blended as a drink. There are several important bioactive compounds present in $C$. asiatica such as triterpene saponins, phenolic compounds, vitamins, minerals, free amino acids, and polyacetylenic compounds [12]. All of these compounds are the major ingredients that are responsible for $C$. asiatica therapeutic effects. It has been denoted in many studies that $C$. asiatica promotes wound healing activity, antimicrobial activity, anticancer activity, antioxidant activity and antileprotic activity [13].

Thus, the aim of this work was to evaluate the effect of various extraction processes, including conventional infusion and decoction and non-conventional ultrasound-assisted extraction (UAE) on the yield and total phenolic content (TPC) of $C$. asiatica aqueous extracts. The antioxidant activity of $C$. asiatica obtained by each extraction technique was also determined.

\section{MATERIALS AND METHODS}

\subsection{Plant Materials and Chemicals}

C. asiatica plants were purchased from local market in Kuantan, Pahang. The plants were washed with running tap water and oven-dried at $50{ }^{\circ} \mathrm{C}$ for three days. The dried plants were ground using a blender to a smaller size before kept in air-tight containers and stored at room temperature until further process. Gallic acid, Folin-Ciocalteu's reagent, 2,2-diphenyl-1-picrylhydrazyl (DPPH), sodium carbonate, sodium nitrate, aluminium chloride, sodium hydroxide, ethanol and methanol were obtained from Sigma Aldrich (St. Louis, US).

\subsection{Extraction Procedure}

Three extraction techniques were studied, which were infusion, decoction and UAE. Infusion was carried out by adding $10 \mathrm{~g}$ of dried C. asiatica to $100 \mathrm{~mL}$ of boiled distilled water and then the mixture was left to stand at room temperature for 30 minutes. While for decoction, $10 \mathrm{~g}$ of dried C. asiatica was boiled with $100 \mathrm{~mL}$ of distilled water for $30 \mathrm{~min}$. The decoction temperature was controlled and maintained at $100{ }^{\circ} \mathrm{C}$. For UAE, $10 \mathrm{~g}$ of dried C. asiatica was mixed with $100 \mathrm{ml}$ of distilled water in a beaker. Extraction was carried out by placing the beaker in an ultrasonic bath (Bandelin Sonorex Digitec, Germany) for $30 \mathrm{~min}$ under the high sonicating setting. Once completed, all the extracts were filtered through Whatman No. 1 filter paper. The filtrates were evaporated by a rotary evaporator (RV8 IKA, Germany) at $40{ }^{\circ} \mathrm{C}$ under reduced pressure for $30 \mathrm{~min}$. The liquid extract was frozen at $-80{ }^{\circ} \mathrm{C}$ for 
four days before proceeded to freeze drying to obtain the powder extract. The samples were stored in a chiller until further analysis.

\subsection{Yield of C. asiatica extract}

The powder obtained from the freeze-drying process was weighed to determine the yield. The percent yield of dried extract was determined by calculating mass of dried extract obtained per mass of propolis used. It was expressed in percent mass/mass.

\subsection{Determination of Total Phenolic Contents}

The total phenolic contents (TPC) of the extracts were determined using Folin-Ciocalteu's reagent, following the method described by Chandra et al. [14] with some modifications. For each sample, 0.5 $\mathrm{mL}$ of the sample was mixed with $3 \mathrm{~mL}$ of distilled water and $0.5 \mathrm{~mL}$ of Folin-Ciocalteu's phenol reagent. After $5 \mathrm{~min}, 1.5 \mathrm{~mL}$ of sodium carbonate solution $(20 \% \mathrm{w} / \mathrm{v})$ was added to the mixture. The mixture was incubated for 2 hours to allow the reaction within the mixture. The absorbance of the samples was measured at $765 \mathrm{~nm}$ using an ultraviolet-visible spectrophotometer (UV-1800 Shimadzu, Japan). The TPC of the extract was calculated based on a standard curve prepared using gallic acid and expressed as mg of gallic acid equivalent (GAE)/g of dry extract. Standard calibration was constructed from different concentrations of gallic acid varying from 25 to $400 \mu \mathrm{g} / \mathrm{mL}$, and its data on absorbance based on calibration equation: $($ Absorbance $)=0.0005[\mathrm{GAE}]+0.0093$ with $\mathrm{R}^{2}=0.97$.

All the determinations were carried out in triplicate.

\subsection{Determination of DPPH Free Radical Scavenging Activity}

Free radical scavenging activity of the extracts was measured using 2,2-diphenyl-1-picrylhydrazyl (DPPH) assay described by Zhao et al. [15] with slight modification. The propolis extracts were dissolved in distilled water to a series of concentration of 25,50 , and $100 \mu \mathrm{g} / \mathrm{mL}$. Briefly, $2 \mathrm{~mL}$ of each sample solution was mixed with $2 \mathrm{~mL}$ of $0.004 \%$ methanolic DPPH solution and incubated in dark for $30 \mathrm{~min}$ at room temperature. Radical scavenging activities were examined by measuring the absorbance of the samples at $517 \mathrm{~nm}$ using an ultraviolet-visible spectrophotometer. The mixture of methanol and DPPH solution was used as a control. All the tests were performed in triplicate and the percentage of scavenging of DPPH free radical was calculated using Equation 1:

Percentage of scavenging of DPPH free radical $=\quad \frac{A_{\text {control }}-A_{\text {sample }}}{A_{\text {control }}} \times 100 \%$

where $A_{\text {sample }}$ is the absorbance of sample and $A_{\text {control }}$ is the absorbance of control (DPPH solution without sample).

\subsection{Statistical Analysis}

All the measurements were performed in triplicate. The results were expressed as mean \pm standard deviation. Statistical analysis was performed using one-way of analysis of variance (ANOVA) by Microsoft Excel Data Analysis Tool 2016. A p-value lower than 0.05 was considered to be statistically significant. 


\section{RESULTS AND DISCUSSION}

\subsection{Effects of Different Extraction Techniques on Yield and Total Phenolic Contents in C. asiatica Extracts}

The effects of different extraction techniques on the yield and TPC of $C$. asiatica extracts are summarized in Table 1. The extraction yields obtained were as varied as the extraction techniques applied. The highest extraction yield was obtained by UAE (25.4\%), followed by decoction (23.6\%) and infusion (18.2\%). Overall, decoction and UAE techniques resulted in higher yield as compared to the infusion technique. For TPC, the amount obtained in $C$. asiatica extracts was between $3.42 \pm 0.030$ to $8.32 \pm 0.105 \mathrm{mg} \mathrm{GAE} / \mathrm{g}$ dry extract. The highest amounts of TPC was obtained by UAE $(8.32 \pm$ $0.105 \mathrm{mg} \mathrm{GAE} / \mathrm{g}$ dry extract), followed by decoction ( $4.77 \pm 0.006 \mathrm{mg}$ GAE$/ \mathrm{g}$ dry extract) and the lowest TPC was shown by infusion $(3.42 \pm 0.030 \mathrm{mg}$ GAE/g dry extract). As reported by Das and Eun [16], extraction via ultrasound-assisted had enhanced the extraction of phytochemicals as compared to conventional extraction techniques. Overall, the TPC obtained is within the range as reported previously [17].

The highest extraction yield and TPC exhibited by the UAE was attributed to the technique that used high intensity and high-frequency sound waves. Propagation and interaction of sound waves to the plant materials disrupted the plant cell walls and enhanced the mass transport of solvent from continuous phase to the plant cells, thus facilitating the release of extractable compounds [18]. Drying and grinding of samples before extraction also assisted in the destruction of cell wall which contributed to higher extraction yield and phytochemicals $[19,20]$.

For conventional extraction techniques like infusion and decoction, the techniques were dependent on the thermal energy. During the extraction process, heat is transferred through convection and conduction [7]. Soaking plant materials in the solvent at elevated temperatures for a specified time could break down the cell walls of the plant. Therefore, a solvent can penetrate through the matrix and the extraction of targeted solute occurred at a more rapid rate. In this study, the time contact between the solvent and plant materials at $100{ }^{\circ} \mathrm{C}$ for the decoction technique was much longer than an infusion. This explained why the decoction technique possesses higher yield and TPC than infusion.

In this work, it can be concluded that the extraction of $C$. asiatica via non-conventional technique, UAE is more efficient than the conventional techniques applied, decoction and infusion. UAE can be used as a plant extraction technique as it has good potential with the highest yield and TPC that provide more elevated health benefits.

Table 1: Extraction yield and TPC of $C$. asiatica extracts obtained using different extraction techniques

\begin{tabular}{lll}
\hline Extraction method & Extraction yield $(\%)$ & TPC \\
& & (mg GAE/g dry extract) \\
\hline Infusion & 18.2 & $3.42 \pm 0.030$ \\
Decoction & 23.6 & $4.77 \pm 0.006$ \\
UAE & 25.4 & $8.32 \pm 0.105$ \\
\hline
\end{tabular}

Note: $\mathrm{GAE}=$ gallic acid equivalent; $\mathrm{TPC}=$ total phenolic contents

3.2 DPPH Radical Scavenging Activity

The antioxidant activities of the $C$. asiatica extracts were evaluated based on the free radical scavenging capacity using DPPH assay as shown in Figure 1. The percentage of DPPH free radical 
inhibition ranged from $75 \%$ to $86 \%$, which varies according to the concentration of the extract. The extract obtained by the UAE technique exhibited the highest radical scavenging activity followed by decoction and infusion at all concentrations of the extract. This result correlated with the TPC evaluated which follows the order of $\mathrm{UAE}>$ decoction > infusion. The regression analysis in Figure 2 shows the correlation between antioxidant activity and TPC in $C$. asiatica extracts. The results indicated a strong association between antioxidant activity and TPC $\left(R^{2}=0.9809\right)$, suggesting that the TPC is probably responsible for the antioxidant activity of $C$. asiatica extracts.

In addition, it was observed that there was a gradual increase in the scavenging effect on the DPPH radical with the increase of extract concentrations from 25 to $100 \mu \mathrm{g} / \mathrm{mL}$. By increasing the concentration of the extracts, it is believed that the phenolic compounds also increased. As reported by Castro-López et al. [9], the amounts of phenolic compounds are correlated with the number of hydroxyl groups available in the reaction medium. Therefore, the possibility of hydrogen donation to free radicals increased. A similar study was reported by Turrini et al. [21], whereby the antioxidant activity exhibited by pomegranate peel extracts correlates with the TPC obtained.

In general, these findings demonstrate that $C$. asiatica extract obtained by UAE possesses the highest free radical scavenging activity. It shows the potential of $C$. asiatica as a source of natural antioxidant. Compared to the conventional methods, UAE is considered as much more promising and has shown a greater potential and better efficiency for the extraction of TPC. Moreover, UAE is cheaper, easy to use and environmental friendly and is not only suitable for laboratory extraction but also pilot and industrial scale.

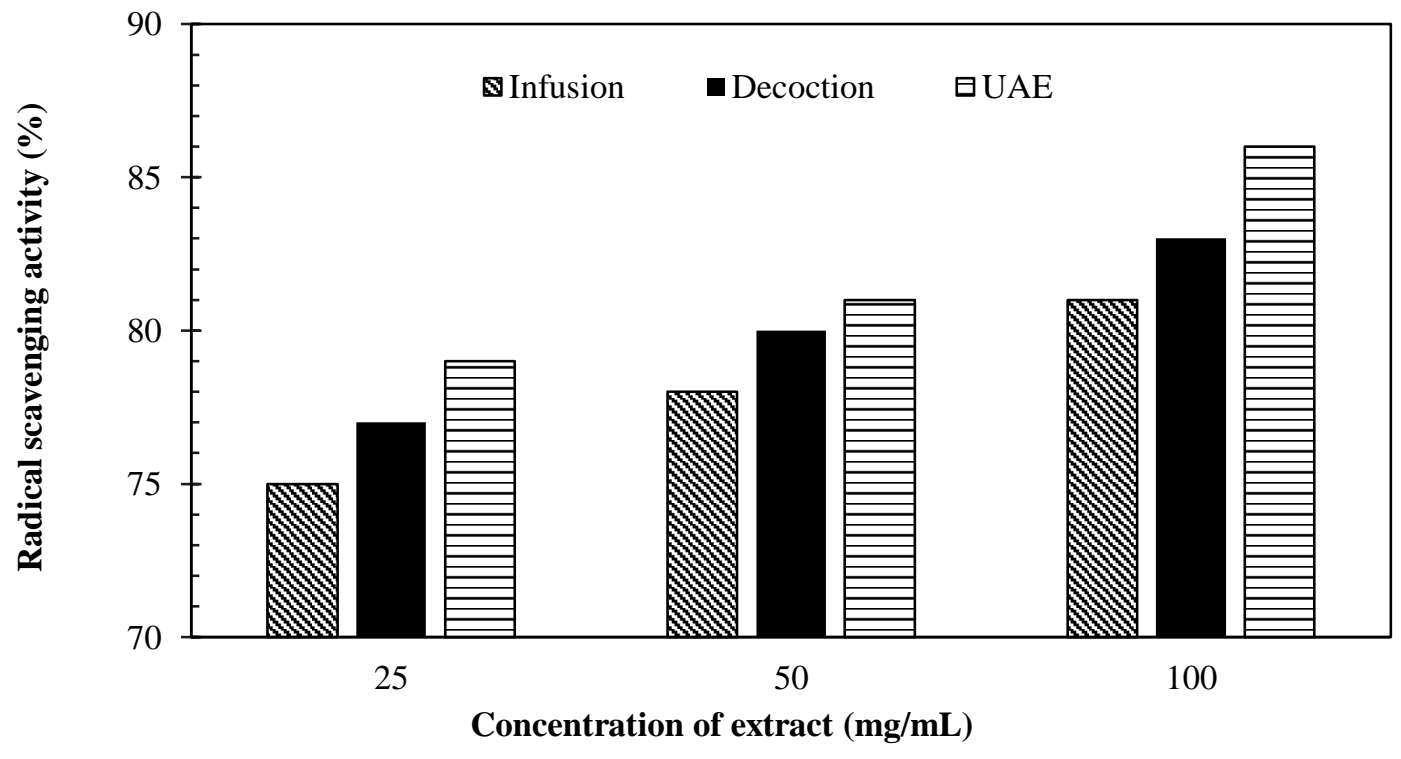

Figure 1: Radical scavenging activity of $C$. asiatica extract. Error bars indicates the standard deviation of means 


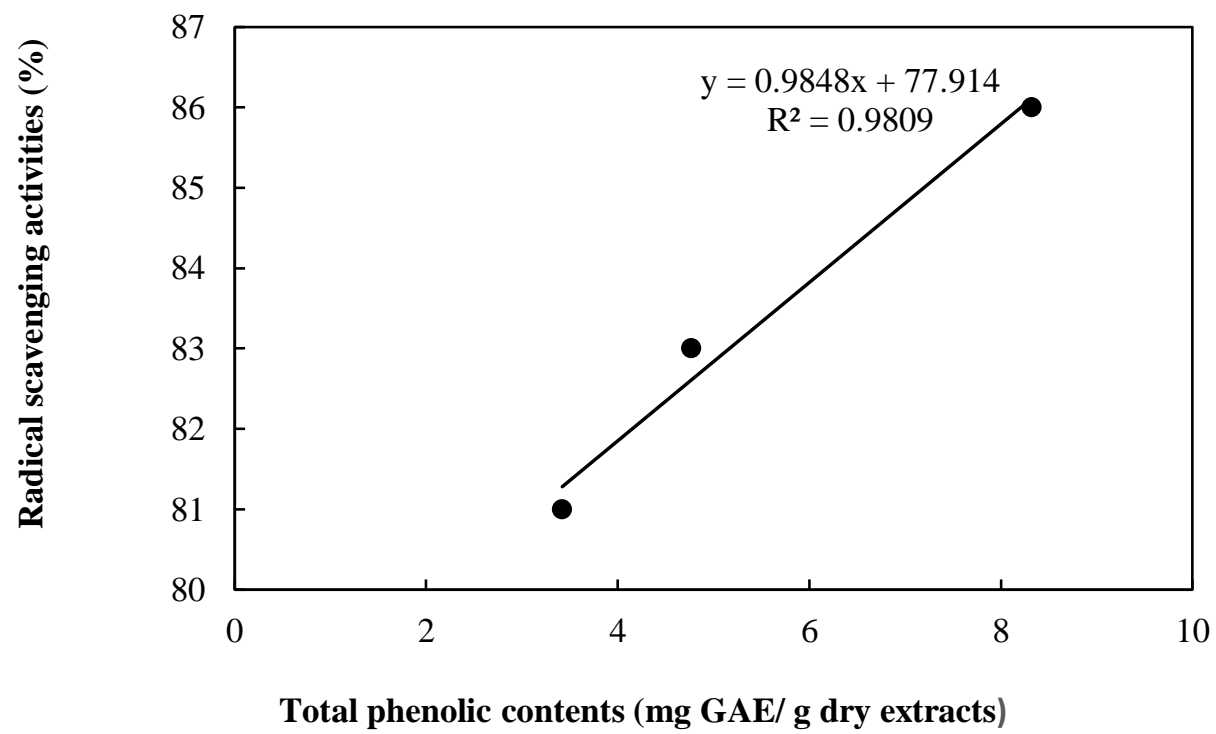

Figure 2: Relationship between total phenolic contents and antioxidant activity of $C$. asiatica extract

\section{CONCLUSIONS}

In the present study, three extraction techniques, namely infusion, decoction and UAE, were studied and compared to obtain the aqueous $C$. asiatica extracts. The results concluded that extraction via UAE is superior to the other extraction techniques studied. The UAE technique has succeeded in enhancing the extraction yield $(25.4 \%)$ compared to infusion (18.2\%) and decoction (23.6\%). Meanwhile, the DPPH assay showed that the antioxidant activities were positively correlated with the TPC obtained. The extract obtained by UAE exhibited the highest TPC $(8.32 \pm 0.105 \mathrm{mg}$ GAE/g dry extract) which contributed to the antioxidant activity, while the infusion $(3.42 \pm 0.030 \mathrm{mg} \mathrm{GAE} / \mathrm{g}$ dry extract $)$ and decoction (4.77 $\pm 0.006 \mathrm{mg}$ GAE/g dry extract) techniques resulted in lower TPC value. This study showed that $C$. asiatica has the potential to be a source of natural antioxidant. Also, UAE is shown to be a simple, viable technique and useful for commercialization of plant extracts. However, comprehensive optimization studies are required for cost reduction, extraction time, energy usage, raw materials and impacts towards the environment.

\section{ACKNOWLEDGEMENTS}

The authors are thankful to the Universiti Malaysia Pahang for providing the funding for this research under UMP Research Grant (RDU1703116). 


\section{REFERENCES}

[1] Guaadaoui, A. "Recent advances in bioactivities of common food biocompoundactives". In Yahia E.M. (Second Edition) Fruit and vegetable phytochemicals: chemistry and human health". John Wiley \& Sons, United Kingdom, 541-594, 2018.

[2] Aghraz, A., Gonçalves, S., Rodríguez-Solana, R., Ait Dra, L., Di Stefano, V., Dugo, G., Cicero, N., Larhsini, M., Markouk, M., and Romano, A. "Antioxidant activity and enzymes inhibitory properties of several extracts from two Moroccan Asteraceae species". South African Journal of Botani, 118: 58-64, 2018.

[3] Albayrak, S., Atasagun, B., and Aksoy, A. "Comparison of phenolic components and biological activities of two Centaurea sp. obtained by three extraction techniques". Asian Pacific Journal of Tropical Medicine, 10: 599-606, 2017.

[4] Biesaga, M. "Influence of extraction methods on stability of flavonoids". Journal of Chromatography A, 1218: 2505-2512, 2011.

[5] Kim, W.J., Kim, J., Veriansyah, B., Kim, J.D., Lee, Y.W., Oh, S.G., and Tjandrawinata, R. "Extraction of bioactive components from Centella asiatica using subcritical water". The Journal of Supercritical Fluids, 48: 211-216, 2009.

[6] Man, F., and Choo, C.Y. "Safety assessment of standardized aqueous Bricea javanica extract in rats". Journal of Ethnopharmacology, 215: 21-36, 2018.

[7] Das, P.R., Kim, Y., Hong, S., and Eun, J.B. "Profiling of volatile and non-phenolic metabolites-amino acids, organic acids, and sugars of green tea extracts obtained by different extraction techniques". Food Chemistry, 296: 69-77, 2019.

[8] da Silva, M.F., Casazza, A.A., Ferrari, P.F., Aliakbarian, B., Converti, A., Bezerra, R.P., Porto, A.L.F., and Perego, P. "Recovery of phenolic compounds of food concern from Arthrospira platensis by green extraction techniques". Algal Research, 25: 391-401, 2017.

[9] Castro-López, C., Ventura-Sobrevilla, J.M., González-Hernández, M.D., Rojas, R., AscacioValdés, J. A., Aguilar, C.N., and Martínez-Ávila, G.C.G. "Impact of extraction techniques on antioxidant capacities and phytochemical composition of polyphenol-rich extracts". Food Chemistry, 237, 1139-1148, 2017.

[10] Mackela, I., Andriekus, T., and Venskutonis, P.R. "Biorefining of buckwheat (fagopyrum esculentum) hulls by using supercritical fluid, Soxhlet, pressurized liquid and enzyme-assisted extraction methods". Journal of Food Engineering, 213: 38-46, 2017.

[11] Subathra S., Shila, S., Devi, M.A., and Panneerselvam, C. "Emerging role of Centella asiatica in improving age related neurological antioxidant status". Experimental Gerontology, 40: 707$715,2015$.

[12] Niamnuy, C., Charoenchaitrakool, M., Mayachiew, P., and Devahastin, S. "Bioactive compounds and bioactivities of Centella asiatica (L.) Urban prepared by different drying methods and conditions". Drying Technology, 31: 2007 - 2015, 2013.

[13] Yasurin, P., Sriariyanun, M., and Phusantisampan, T. "Review: The bioavailability activity of Centella asiatica". KMUTNB International Journal of Applied Science and Technology, 9(1): $1-9,2015$.

[14] Chandra, S., Khan, S., Avula, B., Lata, H., Yang, M.H., ElSohly, M.A., and Khan, I.A. "Assessment of total phenolic and flavonoid content, antioxidant properties, and yield of aeroponically and conventionally grown leafy vegetables and fruit crops: a comparative study". Evidence-based Complementary and Alternative Medicine, 253875, 2014.

[15] Zhao, Y., Chen, S., Wang, Y., Lv, C., Wang, J., and Lu, J. "Effect of drying processes on prenylflavonoid content and antioxidant activity of Epimedium koreanum Nakai". Journal of Food and Drug Analysis, 26: 796 - 806, 2018.

[16] Das, P.R., and Eun, J.B. "A comparative study of ultra-sonication and agitation extraction techniques on bioactive metabolites of green tea extract". Food Chemistry, 253: 22-29, 2018. 
[17] Nirmala, C., Bisht, M.S., Bajwa, H.K., and Santosh, O. "Bamboo: A rich source of natural antioxidants and its application food and pharmaceutical industry". Trends in Food Science \& Technology, 77: 91-99, 2018.

[18] Dhanani, T., Shah, S., Gajbhiye, N.A., and Kumar, S. "Effect of extraction methods on yield, phytochemical constituents and antioxidant activity of Withania somnifera". Arabian Journal of Chemistry, 10: 1193-1199, 2017.

[19] Roshanak, S., Rahimmalek, M., and Goli, S.A.H. "Evaluation of seven different drying treatments in respect to total flavonoid, phenolic, vitamin $\mathrm{C}$ content, chlorophyll, antioxidant activity and color of green tea (Camellia sinensis or C. assamica) leaves". Journal of Food Science and Technology, 53(1): 721-729, 2016.

[20] Zaiter, A., Becker, L., Karam, M.C., and Dicko, A. "Effect of particle size on antioxidant activity and catechin content of green tea powders". Journal of Food Science and Technology, 53(4): 2025-2032, 2016.

[21] Turrini, F., Zunin, P., Catena, S., Villa, C., Alfei, S., and Boggia, R. "Traditional or hydrodiffuion ad gravity microwave coupled with ultrasound as green technologies for the valorization of pomegranate external peels". Food and Bioproducts Processing, 117: 30-37, 2019. 